\title{
Relativistic Transport Approach to Collective Nuclear Dynamics
}

\author{
Serbülent Yildirim \\ Fizik Bölümü, Mersin Üniversitesi, 33343 Mersin, Turkey \\ Theodor Gaitanos \\ Physics Department, Universität München, D-85748 Garching, Germany \\ Massimo Di Toro and Vincenzo Grect* \\ Laboratori Nazionali del Sud, INFN, \\ Via S. Sofia 62, I-95123 Catania, Italy \\ and Dipartimento di Fisica e Astronomia, Universita degli Studi di Catania
}

(Dated: August 5, 2018) 


\begin{abstract}
The isoscalar giant monopole resonance $(I S G M R)$ and isovector giant dipole resonance $(I V G D R)$ in finite nuclei are studied in the framework of a relativistic transport approach. The kinetic equations are derived within an effective nucleon-meson field theory in the Relativistic Mean Field $(R M F)$ scheme, even extended to density dependent vertices. Small amplitude oscillations are analysed using the Relativistic Vlasov $(R V)$ approach, i.e. neglecting nucleon collision terms. The time evolution of the isoscalar monopole moment and isovector dipole moment and the corresponding Fourier power spectra are discussed. The excitation energies of ISGMR and IVGDR are obtained for spherical nuclei with various sets of Lagrangian parameters.

In the case of ${ }^{208} \mathrm{~Pb}$ we study in detail the dependence of the monopole response on the effective mass and symmetry energy at saturation given by the used covariant effective interaction. We show that a reduced $m^{*}$ and a larger $a_{4}$ can compensate the effect on the ISGMR energy centroid of a much larger compressibility modulus $K_{n m}$. This result is important in order to overcome the conflicting determination of the nuclear compressibility between non-relativistic and relativistic effective interactions.

For the symmetry energy dynamical effects, we carefully analyze the influence of the inclusion of an effective isovector scalar channel, $\delta$-meson field, with constant and density dependent couplings. We show that the $\delta$-meson contribution, keeping fixed the equilibrium $a_{4}$ value, leads to a change in the isoscalar and isovector response of finite nuclei which results in a reduction in the centroid energy of $I S G M R$ and $I V G D R$ for ${ }^{208} \mathrm{~Pb}$. All that in fact reveals the relevance of the slope (or pressure) of the symmetry energy at saturation on the ISGMR and IVGDR modes for neutronrich systems.

Density dependent vertices are not much affecting our conclusions. Following as a guidance some extended dispersion relations in nuclear matter, we see two main reasons for that, the smoothness of the density dependences around saturation and the presence of compensation effects coming from rearrangement terms.

PACS numbers: 21.30.Fe, 21.65.+f, 24.30.Cz, 21.10.Re
\end{abstract}

Keywords: Relativistic Transport, Collective Response, Nuclear Compressibility, Symmetry Energy, Effective Hadron Lagrangians. 
*Electronic address: ditoro@lns.infn.it 


\section{INTRODUCTION}

In this work we study the isoscalar giant monopole and isovector giant dipole oscillations in ${ }^{208} \mathrm{~Pb}$ using the Relativistic Vlasov method $(R V)$ in a Relativistic Mean Field $(R M F)$ theory with constant and density dependent meson-nucleon vertices. We analyze various $R M F$ models with different coupling parameters which lead to a different nucleon effective mass $\left(m^{*}\right)$, compressibility modulus $\left(K_{n m}\right)$, symmetry energy $\left(a_{4}\right)$, keeping fixed other nuclear matter saturation properties.

Among those models we focus on the ones where the scalar isovector channel has been introduced by the coupling to an effective $\delta$-meson field [1]. It has been shown that such inclusion has important effects on the equation of state $(E O S)$ and phase diagram of asymmetric nuclear matter $(A N M)$ [1], as well as on the reaction dynamics with exotic nuclei, see the review [2]. In fact the $\delta$-meson brings contributions to the slope and curvature of the symmetry energy and to the neutron-proton effective mass splitting. In particular the influence of this coupling on the collective response of $(A N M)$ appears important, as shown in a linear response approach in ref. [3]. For the comparison of the results we have also considered the well known NL3 [4, 5] parametrization, very successful for finite nuclei structure calculations, and some density dependent vertex models [6, 7] beyond $R M F$. In this way we aim to pin down the sensitivity of isoscalar and isovector collective oscillations on $\mathrm{m}^{*}$, $K_{n m}$ and $a_{4}$, focussing on the interplay between isoscalar-isovector couplings. In particular we will see that the covariant structure of the isovector part of the effective interaction will clearly show up even in the isoscalar monopole mode in ${ }^{208} \mathrm{~Pb}$.

Relativistic mean field models, with constant [8, 9] and density dependent [10] mesonnucleon couplings, have been applied to the description of collective excitations of atomic nuclei in the framework of Time-Dependent Relativistic Mean Field (TDRMF) and in the self-consistent relativistic random phase approximation $(R R P A)$. The monopole predictions of nonrelativistic Hartree-Fock plus random phase approximation ( $R P A$ ) calculations, using both Skyrme and Gogny effective interactions, seem to indicate that the value of $K_{n m}$ should be in the range $210-220 \mathrm{MeV}$ [11, 12]. In relativistic mean-field models on the other hand, results of both time-dependent and $R P A$ calculations suggest that empirical $G M R$ energies are best reproduced by an effective force with $K_{n m}$ 250-270 MeV [13, 14]. This difference has been pointed out as a serious open problem [10]. A possible solution to this ambiguity was 
suggested in Ref.[3] as an interplay between the effective mass and compressibility within a discussion of isoscalar response of nuclear matter. Here we show the validity of this interpretation, joint to a density dependence of the symmetry energy, also for the spherical finite n-rich nuclei, like ${ }^{208} \mathrm{~Pb}$. Recent studies are in fact pointing to the same isospin effect, see refs. [15, 16, 17]. In this respect we have even analyzed the influence of the inclusion of the $\delta$-meson on both isoscalar and isovector response in spherical n-rich nuclei.

The introduction of density dependent vertices, following the Dirac - Brueckner Hatree - Fock estimations, is not showing relevant new effects. A nice analitical interpretation of this result, based on a linear response theory for nuclear matter, is finally presented.

\section{OUTLINE OF THE MODEL}

The dynamics of collective vibrations in spherical nuclei is studied in the framework of the relativistic Vlasov $(R V)$ transport equation, which describes the dynamical evolution of a semi-classical phase space distribution function $f(x, p)$ under the influence of the nuclear mean field. Binary collisions between nucleons will be not considered here. Thorough derivations of the $R V$ transport equations from an effective hadron-meson field theory, 18] can be found elsewhere [19, 20, 21]. The $R V$ equation reads $(i=p, n)$ :

$$
\left[p_{i}^{* \mu} \partial_{\mu}+\left(p_{\nu i}^{*} \mathcal{F}_{i}^{\mu \nu}+m_{i}^{*} \partial^{\mu} m_{i}^{*}\right) \partial_{\mu}^{\left(p_{*}\right)}\right] f_{i}\left(x, p^{*}\right)=0
$$

whith the field tensor

$$
\mathcal{F}_{i}^{\mu \nu} \equiv \partial^{\mu} \Sigma_{i}^{\nu}-\partial^{\nu} \Sigma_{i}^{\mu}
$$

and effective masses and kinetic momenta $m^{*}$ and $p^{* \mu}$, respectively, specified below. The particles obey the mass-shell condition

$$
p_{i}^{* \mu} p_{i \mu}^{*}-m_{i}^{* 2}=0
$$

Thus, from the temporal knowledge of the phase space distribution function one can calculate the time evolution of physical quantities such as densities and fields. We remind the meaning of the the Wigner matrix (in the Spinor space) 


$$
\begin{aligned}
F_{\alpha \beta}(x, p) & =<\hat{F}_{\alpha \beta}(x, p)> \\
& \equiv \frac{1}{(2 \pi)^{4}} \int d^{4} R e^{-i p_{\mu} R^{\mu}}<\bar{\psi}_{\beta}\left(x+\frac{1}{2} R\right) \psi_{\alpha}\left(x-\frac{1}{2} R\right)>
\end{aligned}
$$

¿From the above definition it follows that single-particle operators can be expressed as (spinor indices will be omitted for simplicity)

$$
<\hat{O}>=\int d^{4} x \int d^{4} p \operatorname{tr}(\hat{O} F(x, p))
$$

where the trace runs over spin and isospin indices. The scalar density and the vector current, for example, assume the form

$$
\begin{aligned}
& \rho_{s}(x)=<\bar{\psi} \psi>=\int d^{4} p \operatorname{tr}(F(x, p)) \\
& j_{\mu}(x)=<\bar{\psi} \gamma_{\mu} \psi>=\int d^{4} p \operatorname{tr}\left(\gamma_{\mu} F(x, p)\right),
\end{aligned}
$$

and will be used to calculate the different Lorentz components of the mean field potential.

The nuclear mean field $U$ is characterized in a $R M F$ theory by means of self energies in the form $U=\Sigma_{s}-\gamma_{\mu} \Sigma^{\mu} \ldots$ (higher contributions are usualy neglected due to symmetry properties of nuclear matter). In a Non-Linear $(Q H D-N L)$ model with isoscalar scalar and vector meson fields $\sigma$ and $\omega$ and with the inclusion of the isovector channel through the exchange of the virtual charged $\delta$ (scalar) and $\rho$ (vector) mesons, the mean field approximation leads to self energies which are related to the expectation values of the combination of isoscalar and isovector fields with coupling constants $g_{\sigma}, g_{\omega}, g_{\rho}$ and $g_{\delta}$. The scalar and vector components of the self energies are generally given by

$$
\begin{gathered}
\Sigma_{i}^{\mu}=g_{\omega} \omega^{\mu}(x) \pm g_{\rho} b^{\mu}(x) \quad\left\{\begin{array}{l}
+\operatorname{proton}(i=p) \\
-\operatorname{neutron}(i=n)
\end{array}\right. \\
\Sigma_{s i}=g_{\sigma} \sigma(x) \pm g_{\delta} \delta(x) \quad\left\{\begin{array}{l}
+\operatorname{proton}(i=p) \\
-\operatorname{neutron}(i=n)
\end{array}\right.
\end{gathered}
$$

with the expectation values of the fields self-consistently calculated, see later Eqs.(19).

The self energies characterize the in-medium properties of the nucleons inside the hadronic environment in terms of kinetic momenta and effective masses

$$
p_{i}^{* \mu}=p_{i}^{\mu}-\Sigma_{i}^{\mu}
$$




$$
m_{i}^{*}=m-\Sigma_{s i}
$$

The density dependence of the mean field, i.e. the density behavior of the self energies, depends on the coupling choices of the $R M F$ model. Here we consider different parametrizations within the Non-Linear $(Q H D-N L)$ effective field approach and even extended to the Density Dependent Hadronic $(D D H)$ mean field theory [6, 7, 22]. In table [1 the values for the different coupling constants and the non-linear parameters for different sets of NonLinear Walecka $(Q H D-N L)$ models are presented, for details see refs. [1, 2, 3, 4, 5, 7]. Their corresponding nuclear matter saturation properties are given in table II. In the $D D H$ models the density dependence of the coupling constants is chosen in order to reproduce microscopic Dirac - Brueckner - Hartree - Fock results beyond the RMF picture, see the discussion in ref. [22].

The choice of models with rather different nuclear matter properties has been done on purpose, in order to investigate the role of the effective masses, compressibility and symmetry energy on isoscalar (monopole) and isovector (dipole) oscillations in neutron-rich nuclei. We have compared various Non-Linear $R M F$ parametrizations, in particular the Giessen sets NL1 - G, NL2-G, 23], extended also to simulations of relativistic heavy ion collisions, and the widely used NL3 set [4, 5], successfully applied in finite nuclei studies. The different treatment of the iso-vector part of the mean field (competition effects of the repulsive $\rho$ field and the attractive $\delta$ meson) is analysed in detail using the $(N L \rho, N L \rho \delta)$ parameter sets 1]. The same analysis is performed within density dependent coupling models, the parametrizations $D D H 3 \rho$ and $D D H 3 \rho \delta$ of ref. 22] have been used.

The dynamics of collective vibrations in spherical nuclei is simulated in the framework of the relativistic Vlasov equation (11). Its numerical realization is based on the standard test particle method, where the phase space distribution function $f(x, p)$ is represented by a finite number of test particles of a covariant Gaussian form (Relativistic Landau-Vlasov $(R L V)$ method). A detailed description of the $R L V$ method is given in Ref. [24], here this model will be briefly discussed. The use of a Gaussian shape for the test particles has the advantage of smooth distribution functions, but maintaining an accurate determination of local quantities, particularly important near the nuclear surface.

The covariant Gaussians in the four-dimensional Minkowski space are defined as 
TABLE I: Coupling parameters in terms of $f_{i} \equiv\left(g_{i} / m_{i}\right)^{2}$ for $i=\sigma, \omega, f_{i} \equiv\left(g_{i} / 2 m_{i}\right)^{2}$ for $\rho, \delta$, $A \equiv a / g_{\sigma}^{3}$ and $B \equiv b / g_{\sigma}^{4}$ for various Non-Linear $R M F$ models using the $\rho$ and both $\rho$ and $\delta$ mesons for the characterization of the isovector mean field. In the $D D H$ models the coupling functions are explicitly density dependent [6, 7, 22].

\begin{tabular}{cccccc}
\hline \hline Parameter & NL1-G & NL2-G & NL3 & $N L \rho$ & $N L \rho \delta$ \\
\hline$f_{\sigma}\left(f m^{2}\right)$ & 6.146 & 9.300 & 15.739 & 10.330 & 10.330 \\
$f_{\omega}\left(f m^{2}\right)$ & 3.611 & 3.611 & 10.530 & 5.420 & 5.420 \\
$f_{\rho}\left(f m^{2}\right)$ & 1.200 & 1.220 & 1.339 & 0.950 & 3.150 \\
$f_{\delta}\left(f m^{2}\right)$ & 0.0 & 0.0 & 0.0 & 0.0 & 2.500 \\
$A\left(f m^{-1}\right)$ & -0.123 & 0.0824 & -0.01 & 0.033 & 0.033 \\
$B$ & 0.169 & -0.0012 & -0.003 & -0.0048 & -0.0048 \\
\hline \hline
\end{tabular}

TABLE II: Nuclear matter saturation properties in the different $R M F$ models.

\begin{tabular}{cccccc}
\hline \hline Property & NL1-G & NL2-G & NL3 & $N L \rho, \rho \delta$ & $D D H \rho, \rho \delta$ \\
\hline$E / A(\mathrm{MeV})$ & -16.0 & -16.0 & -16.3 & -16.0 & -16.0 \\
$\rho_{0}\left(\mathrm{fm}^{-3}\right)$ & 0.145 & 0.145 & 0.148 & 0.160 & 0.153 \\
$m^{*} / m$ & 0.83 & 0.83 & 0.60 & 0.75 & 0.55 \\
$K_{n m}(\mathrm{MeV})$ & 380 & 210 & 272 & 240 & 240 \\
$a_{4}(\mathrm{MeV})$ & 30.62 & 30.62 & 37.40 & 30.50 & 33.40 \\
\hline \hline
\end{tabular}

$$
\begin{aligned}
G\left(x ; \xi_{i}\right) & \equiv \int_{-\infty}^{-\infty} d \tau g\left(x-x_{i}(\tau)\right) \\
& =\alpha \int_{-\infty}^{-\infty} d \tau \exp \left(\left(x-x_{i}(\tau)\right)^{2} / w^{2}\right) \delta\left[\left(x_{\mu}-x_{i \mu}(\tau)\right) u_{i}^{\mu}(\tau)\right]
\end{aligned}
$$

where $\xi_{i}$ denotes the world line of the particle $i$ as a whole, $\tau$ refers to the eigentime of the test particle and $\alpha$ is the normalization constant. In the four-dimensional momentum space a gaussian weight of a test particle is defined by [24]

$$
g\left(p^{*}-p_{i}^{*}(\tau)\right) \equiv \alpha_{p} \exp \left(\left(p^{*}-p_{i}^{*}(\tau)\right)^{2} / w_{p}^{2}\right) \delta\left[p_{\mu}^{*} p_{i}^{* \mu}(\tau)-m_{i}^{* 2}\right]
$$

where the center of the gaussian is assumed to be on-shell i.e. $p_{i \mu}^{*}=m_{i}^{*} u_{i \mu}, u_{i}^{2}=1$, whereas the free momentum $p_{\mu}^{*}$ is generally off-shell. The effective mass of the particle is taken as 
$m_{i}^{*}=m^{*}\left(x_{i}(\tau)\right)$. The norm of the gaussian is calculated in the rest frame of the particle to be $m_{i}^{*-1}$ with $\alpha_{p}=\left(\sqrt{\pi} w_{p}\right)^{-3}$, where $w$ and $w_{p}$ are the test particle widths in position and momentum space.

With the Gaussians of (12 13) the phase space distribution function $f\left(x, p^{*}\right)$ is expressed as

$$
\begin{aligned}
f\left(x, p^{*}\right)= & \frac{1}{N} \sum_{i=1}^{A \cdot N} \int_{-\infty}^{+\infty} d \tau g\left(x-x_{i}(\tau)\right) g\left(p^{*}-p_{i}^{*}(\tau)\right) \\
= & \frac{1}{N\left(\pi w w_{p}\right)^{3}} \sum_{i=1}^{A \cdot N} \int_{-\infty}^{-\infty} d \tau \exp \left(\left(x-x_{i}(\tau)\right)^{2} / w^{2}\right) \\
& \times \exp \left(\left(p^{*}-p_{i}^{*}(\tau)\right)^{2} / w_{p}^{2}\right) \\
& \times \delta\left[\left(x_{\mu}-x_{i \mu}(\tau)\right) u_{i}^{\mu}(\tau)\right] \delta\left[p_{\mu}^{*} p_{i}^{* \mu}(\tau)-m_{i}^{* 2}\right]
\end{aligned}
$$

where $N$ is the number of test particles per nucleon. Scalar densities $\rho_{s}$ and baryon currents $j_{\mu}$ follow from the phase space distribution

$$
\begin{aligned}
& \rho_{s}(x)=\frac{1}{N} \sum_{i=1}^{A \cdot N} \int_{-\infty}^{-\infty} d \tau \frac{m^{*}(x)}{m^{*}\left(x_{i}(\tau)\right)} g\left(x-x_{i}(\tau)\right) \\
& j_{\mu}(x)=\frac{1}{N} \sum_{i=1}^{A \cdot N} \int_{-\infty}^{-\infty} d \tau g\left(x-x_{i}(\tau)\right) u_{i \mu}(\tau) .
\end{aligned}
$$

The equations of motion for the test particle trajectories are given by

$$
\begin{aligned}
\frac{d}{d \tau} x_{i}^{\mu}(\tau)= & u_{i}^{\mu}(\tau) \\
\frac{d}{d \tau} u_{i}^{\mu}(\tau)= & \frac{1}{m^{*}\left(x_{i}\right)} \sum_{j=1}^{A \cdot N} \frac{2}{w^{2}}\left[\frac{g_{\omega}^{2}}{m_{\omega}^{2}} u_{i \nu}\left(R_{j}^{\mu}\left(x_{i}\right) u_{j}^{\nu}-R_{j}^{\nu}\left(x_{i}\right) u_{j}^{\mu}\right)\right. \\
& \left.-g_{\sigma} \frac{\partial \sigma\left(x_{i}\right)}{\partial \rho_{s}}\left(R_{j}^{\mu}\left(x_{i}\right)-u_{i}^{\mu} u_{i}^{\nu} R_{j \nu}\left(x_{i}\right)\right)\right] \frac{\exp \left(R_{j}^{2}\left(x_{i}\right) / w^{2}\right)}{N(\sqrt{\pi} w)^{3}} \\
\pm & \frac{1}{m^{*}\left(x_{i}\right)} \frac{2}{w^{2}} \sum_{j=1}^{Z \cdot N}\left[\frac{g_{\rho}^{2}}{4 m_{\rho}^{2}} u_{i \nu}\left(R_{j}^{\mu}\left(x_{i}\right) u_{j}^{\nu}-R_{j}^{\nu}\left(x_{i}\right) u_{j}^{\mu}\right)\right. \\
& \left.-\frac{g_{\delta}^{2}}{4 m_{\delta}^{2}} u_{i \nu}\left(R_{j}^{\mu}\left(x_{i}\right)-u_{i}^{\mu} u_{i}^{\nu} R_{j \nu}\left(x_{i}\right)\right)\right] \frac{\exp \left(R_{j}^{2}\left(x_{i}\right) / w^{2}\right)}{N(\sqrt{\pi} w)^{3}} \\
\mp & \frac{1}{m^{*}\left(x_{i}\right)} \frac{2}{w^{2}} \sum_{j=Z \cdot N+1}^{A \cdot N}\left[\frac{g_{\rho}^{2}}{4 m_{\rho}^{2}} u_{i \nu}\left(R_{j}^{\mu}\left(x_{i}\right) u_{j}^{\nu}-R_{j}^{\nu}\left(x_{i}\right) u_{j}^{\mu}\right)\right. \\
& \left.-\frac{g_{\delta}^{2}}{4 m_{\delta}^{2}} u_{i \nu}\left(R_{j}^{\mu}\left(x_{i}\right)-u_{i}^{\mu} u_{i}^{\nu} R_{j \nu}\left(x_{i}\right)\right)\right] \frac{\exp \left(R_{j}^{2}\left(x_{i}\right) / w^{2}\right)}{N(\sqrt{\pi} w)^{3}}
\end{aligned}
$$


with

$$
R_{i}^{\mu}(x) \equiv\left(x^{\mu}-x_{i}^{\mu}(\tau)\right)-\left(x_{\nu}-x_{i \nu}(\tau)\right) u_{i}^{\nu}(\tau) u_{i}^{\mu}(\tau)
$$

projection of the vector $\left(x-x_{i}(\tau)\right)$ on the hyperplane perpendicular to $\left.u_{i}(\tau), 24\right]$.

Here the equations for velocities, rather than for momenta, are given, within the assumption that the particle accelerations are small. In this equation particles are propagated by their respective eigentimes and so corresponding time coordinates $x_{i 0}(\tau)$ can differ. In order to solve the problem of different time coordinates a system time has been adopted for the propagation [24].

The scalar and vector meson fields, determined by the scalar density $\rho_{s}$ and the baryonic current $j_{\mu}$, respectively, result from the solution of the corresponding equations in the Local Density Approximation $(L D A)$

$$
\begin{aligned}
m_{\sigma}^{2} \sigma(x)+B \sigma^{2}(x)+C \sigma^{3}(x) & =g_{\sigma} \rho_{s}(x) \equiv g_{\sigma} \int d^{4} p^{*} \frac{m^{*}(x)}{E^{*}(x)} f\left(x, p^{*}\right) \\
\omega_{\mu}(x) & =\frac{g_{\omega}}{m_{\omega}^{2}} j_{\mu}(x) \equiv \frac{g_{\omega}}{m_{\omega}^{2}} \int d^{4} p^{*} p_{\mu}^{*} f\left(x, p^{*}\right) \\
b_{\mu}(x) & =\frac{g_{\rho}}{4 m_{\rho}^{2}} j_{3 \mu}(x) \equiv \frac{g_{\rho}}{4 m_{\rho}^{2}} \int d^{4} p^{*} p_{\mu}^{*} f_{3}\left(x, p^{*}\right) \\
\delta(x) & =\frac{g_{\delta}}{4 m_{\delta}^{2}} \rho_{s 3}(x) \equiv \frac{g_{\delta}}{4 m_{\delta}^{2}} \int d^{4} p^{*} \frac{m^{*}(x)}{E^{*}(x)} f_{3}\left(x, p^{*}\right) .
\end{aligned}
$$

with $f_{3} \equiv f_{p}-f_{n}$.

An important issue for the description of low energy excitations within the $R V$ equation is an appropriate initialization of finite nuclei, before starting the phase space evolution of the distribution function with Eq. (18). The ground state of a spherical nucleus is randomly initialized by means of the test particles with the covariant Gaussian shape in position and momentum space. After the first step of a randomly obtained initial distribution, a fit procedure in coordinate space is performed according to realistic Thomas-Fermi reference density distributions. During this procedure the proton and neutron distributions are fitted separately by satisfying the empirical values for the asymmetry and the surface thickness parameters, respectively. In the Thomas-Fermi calculation the scalar densities are determined by solving self-consistently the equations for the effective masses $m_{p, n}^{*}$. With this initialization of a given spherical nucleus the temporal evolution is described by the $R L V$ equation (18). We have used 100 test particles per nucleon for the transport descriptions, 
which yields a smooth distribution function with a very good energy conservation.

For giant resonances the time dependence of collective dynamical quantities is not periodic, since giant resonances are generally not stationary states of the mean-field Hamiltonian 9]. For non-relativistic models it has been proven that the results from the Vlasov equation are identical to results from full quantum mechanical calculations $(T D H F)$ 25]. Since in the non-relativistic frame $R P A$ is the small amplitude limit of a $T D H F$ calculation, one can expect that the results from the relativistic Vlasov equation are comparable to those from relativistic $R P A$ calculations of the type discussed in Ref. [13]. In the small amplitude limit the energy obtained from the frequency of the oscillation coincides with the experimental energy of the collective oscillation.

The collective dynamical variables that characterize nuclear vibrations are defined as expectation values of single particle operators in the phase space representation. For the isoscalar monopole vibrations, the time dependent monopole moment is defined as

$$
\left\langle r^{2}(t)\right\rangle=\frac{1}{A} \int d^{3} x r^{2} j_{0}(x) .
$$

In this work we have applied the $R L V$ method to isoscalar monopole and isovector dipole oscillations in ${ }^{208} \mathrm{~Pb}$. The excitation of an iso-scalar monopole oscillation of the initialized nucleus in its ground state is modeled by a radial expansion. This is done by introducing a new coordinate

$$
r_{\text {mon }}=(1+a) r
$$

for each test particle. Here $a$ is a scaling parameter, $a=0.1 \mathrm{fm}$ has been used, and the deformation of protons and neutrons is in phase.

For the isovector dipole oscillation the following operator has been applied [27]

$$
\hat{Q}_{1 \mu}^{T=1}=\frac{N}{N+Z} \sum_{p=1}^{z} r_{p} Y_{1 \mu}-\frac{Z}{N+Z} \sum_{n=1}^{N} r_{n} Y_{1 \mu} .
$$

This means an out of phase shift along the z-axis between protons and neutrons. We have used a scaling parameter of $1 \mathrm{fm}$ according to Eq.(22), which causes a center of mass separation between protons and neutrons, while keeping unchanged the center of mass of the whole system. 


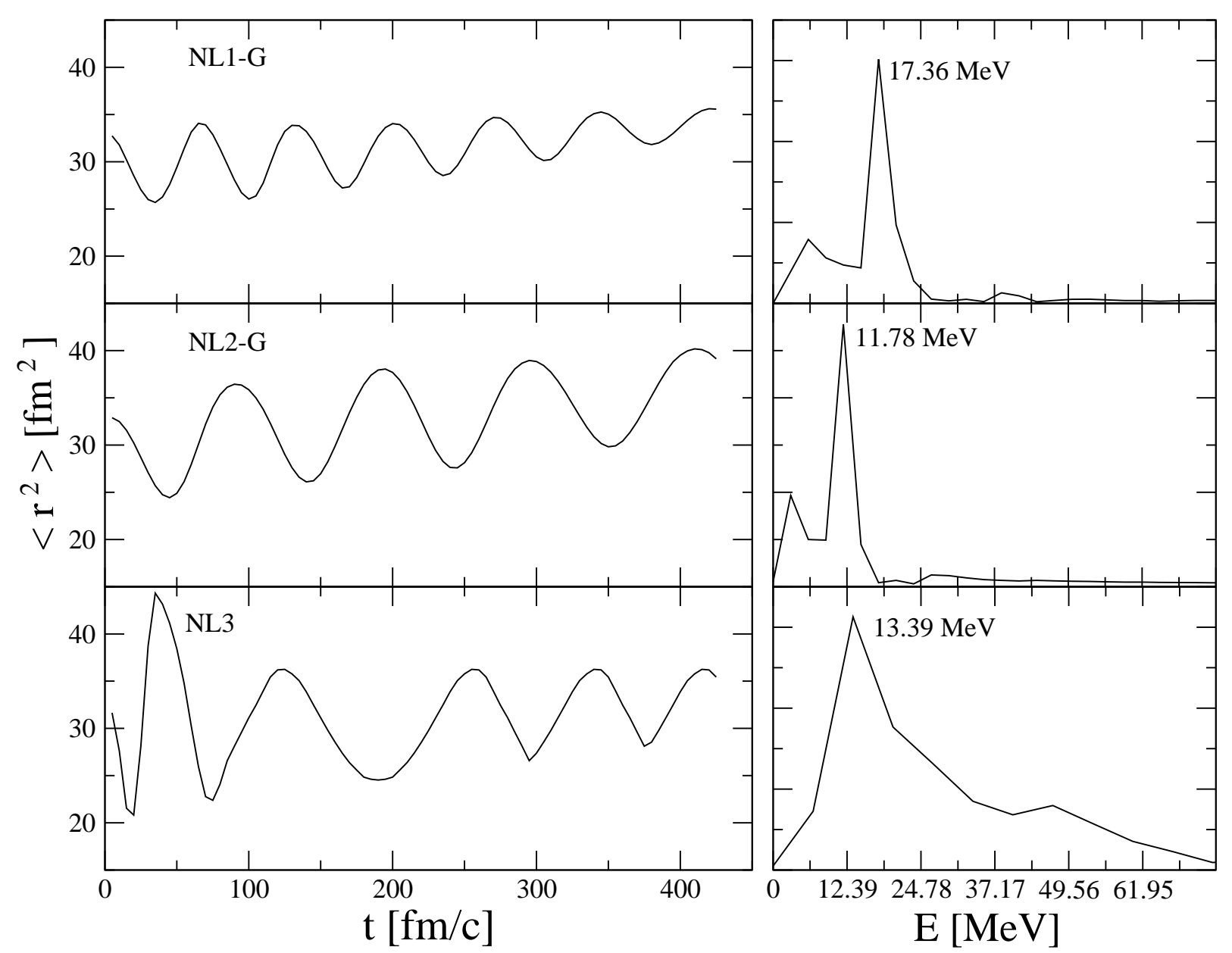

FIG. 1: (Left) Time-dependent isoscalar $\left\langle r^{2}\right\rangle$ monopole moment and (right) the corresponding Fourier power spectra for ${ }^{208} \mathrm{~Pb}$, as obtained from transport calculations with different choices of the nuclear mean field: ( $N L 1-G, N L 2-G, N L 3)$ non-linear parametrizations of Refs. [4, 23], respectively. The corresponding excitation energies of the $I S G M R$ are indicated in the right pannels.

\section{ISOSCALAR MONOPOLE OSCILLATIONS}

The study of Isoscalar Giant Monopole Resonances, ISGMR, in nuclei provides an important source of information on the nuclear matter compressibility. The complete experimental data set on isoscalar monopole resonances has been analyzed by Shlomo and Youngblood [26]. In fact, within a semi-empirical macroscopic approach of the systematics of $G M R$, it turns out that starting from finite nuclei data the nuclear matter compression modulus $K_{n m}$ can be only fixed within the rather wide range $200-350 \mathrm{MeV}$. A systematic theoretical 
work, based on non-relativistic RPA methods with realistic effective interactions [12], was supporting the lower region of the above range, around $210-220 \mathrm{MeV}$. At variance relativistic approaches, within the same small amplitude limits, were pointing to some definitely larger values, $250-270 \mathrm{MeV}$ [10]. We address the problem studying in a relativistic frame the $I S G M R$ in ${ }^{208} \mathrm{~Pb}$, that shows a well established Giant Monopole Resonance (GMR) at $13.7 \pm 0.3 \mathrm{MeV}$. Moreover ${ }^{208} \mathrm{~Pb}$ is interesting since it represents a neutron-rich nucleus, where we can even reveal isovector effects.

As discussed in the previous section, the iso-scalar monopole oscillations are analyzed within the relativistic Vlasov transport equation. The nuclear mean field is evaluated in the Non-Linear versions of the Walecka model and in the DDH approach, see Tables \and 【. We will discuss the dependence of the $G M R$ on the compression modulus, e.g. by comparing the Giessen parametrizations (NL1-G and NL2-G) with a similar value for $m^{*}$ but different $K_{n m}$, and on the effective mass (for fixed $K_{n m}$ ), e.g. by comparing the parametrizations of Liu et al. with the $D D H$ model. The latter will allow also some comments on the effect of a density dependence of the couplings around the saturation point. These analyses are comparable with similar studies in the framework of a time dependent relativistic mean field model, see Refs. [4, 13, 29], and within non-relativistic RPA calculations of the nuclear matter compressibility using Gogny effective interactions, see Ref. [12].

The influence of a different treatment of the iso-vector part of the mean field will be particularly discussed either paying attention to the $a_{4}$ differences of the various interactions, see Table II or more specifically focussing on the effects of the iso-vector, scalar $\delta$ field, i.e. by comparing the results of parametrizations, like $N L \rho, N L \rho \delta$ and $D D H \rho, D D H \rho \delta$, with and without the introduction of the $\delta$ meson. We note again that the inclusion of the $\delta$ meson in the interaction leads to a stiffer symmetry energy (around saturation) and to a splitting between the (Dirac) effective masses of neutrons and protons, see a review in ref. [2]. Also the vector component of the iso-vector self energy is modified due to the enhancement of the vector, iso-vector $\rho$ meson field roughly by a factor of 3 .

Fig. 1 shows the dynamical evolution of the monopole moment of the excited ${ }^{208} \mathrm{~Pb} \mathrm{nu}-$ cleus for those models with fixed effective mass $(N L 1-G, N L 2-G)$ and different compression modulus. The ISGMR oscillation is modeled by Eq. (201). The numerical simulation of the $R V$ equation is performed with the test particle method of the previous section. The limited number of test particles per nucleon (100 in our case) leads to numerical fluctuations, 
which can be seen in Fig. 1 in terms of spurious oscillations with very low frequencies (first peaks in the Fourier energy spectra) and in terms of a partially non-periodic evolution of the monopole moment. This is a general non-trivial feature of transport descriptions with test particle methods which leads to an artificial damping of the excitation [30]. However, due to a rather good stability of the nucleus (until several $100 \mathrm{fm} / \mathrm{c}$ ) and a good separation of the ambigous excitation energy of the numerical oscillations, we conclude on the reliability of the results presented in the following.

The excitation energy of the ISGMR oscillation is very sensitive to the nuclear matter compressibility, which is a well known fact. In particular, the excitation energy increases with rising compression modulus (from $N L 2-G$ to $N L 1-G)$ ), as expected.

The experimental value of $13.7 \pm 0.3 \mathrm{MeV}$ can be reproduced satisfactory by the $N L 3$ model $(13.39(\hbar \omega))$. This result is consistent with the analysis of Ref. [4], again as an important check of the transport calculations.

We notice however that the $N L 2-G$ model, with $K_{n m}=210 \mathrm{MeV}$, leads to a centroid excitation energy $(11.78(\hbar \omega))$ not too far from that of the $N L 3$ model, which has a much larger compressibility, $K_{n m}=272 \mathrm{MeV}$. Considering the other saturation properties of the NL3 force, see Table II this fact is pointing to two interesting implications: i) The dependence of the monopole frequency on the effective nucleon mass; ii) The dependence on the symmetry energy, the $a_{4}$ parameter, that one could expect since the ${ }^{208} \mathrm{~Pb}$ is a neutronrich system. We will discuss in detail the two effects in the following. We note that, as a straightforward consequence, the claimed discrepancy on the nuclear matter compressibility modulus $K_{n m}$ between relativistic and non-relativistic models, see [10], can be eventually overcome.

\section{Monopole frequency and effective mass}

A linear response theory for nuclear matter within the $R M F$ frame can give some interesting hints. We can derive a dispersion relation, see refs. [2, 3],

$$
1+\frac{E_{F}^{*}}{3 k_{F}^{2}}\left[K_{n m}^{p o t}-9 f_{\omega} \frac{k_{F}^{2}}{E_{F}^{* 2}}\left(1-f_{\sigma} \frac{m^{*}}{E_{F}^{* 2}} \rho_{S}\right) \rho_{B}\right] \varphi(s)=0
$$


where $s$ is the dispersion parameter $s \equiv \frac{v_{s}}{v_{F}}=\frac{\omega}{k \cdot v_{F}}$ and $\varphi(s)$ is the usual Lindhard function of the Landau Fermi Liquid theory:

$$
\varphi(s)=1-\frac{s}{2} \ln \left|\frac{s+1}{s-1}\right|+\frac{i}{2} \pi s \theta(1-s)
$$

Here the $K_{n m}^{p o t}$ is the potential part of the nuclear matter compressibility.

From Eq.(23) we see that the "restoring" force for monopole oscillations is given by an effective compressibility which is reduced for larger values of the $\omega$ meson coupling constant. However $f_{\omega}$ can assume very different values depending on the chosen value for effective masses $m^{*}$. This is easy to understand since in the $R M F$ limit the saturation binding energy has the simple form

$$
E / A\left(\rho_{0}\right)=E_{F}^{*}+f_{\omega} \rho_{0}-m_{N}
$$

where $m_{N}$ is the bare nucleon mass. Thus in order to have the same saturation values of $\rho_{0}$, $E / A\left(\rho_{0}\right)$, when we decrease $m^{*}$ we have to increase $f_{\omega}$. We then come to the natural conclusion that two EOS with different effective masses at saturation, even if the compressibilities are the same, are expected to have different dynamical monopole response. In the $N L G-2$ vs. NL3 comparison discussed here we clearly see the interplay between compressibility and effective mass: we can get similar monopole energies increasing the compressibility while decreasing the effective mass, see Table II.

This appears to be a quite general feature, present also in non-relativistic approaches, see the Fig.7 of the ref. [12] where the RPA systematics of the Gogny forces is shown: the ${ }^{208} \mathrm{~Pb}$ breathing mode energy is not much changing if at the same time we increase the $N M$ compressibility and decrease the effective mass. A similar trend has been suggested in a recent work on the nuclear compressibility within the nonrelativistic frame 17].

\section{Monopole frequency and symmetry energy}

It is well known that the equilibrium properties of nuclear matter are changing with isospin asymmetry, in particular the saturation density and the corresponding EoS curvature , see [2], Sect.2 and refs. therein. For the compressibility shift we have, after some algebra:

$$
\begin{array}{r}
\Delta K_{n m}(I)=\left.9 \rho_{0}\left[\rho_{0} \frac{d^{2}}{d \rho^{2}}-2 \frac{d}{d \rho}\right] \epsilon_{\text {sym }}(\rho)\right|_{\rho=\rho_{0}} I^{2} \\
=\left[K_{\text {sym }}-6 L\right] I^{2}<0,
\end{array}
$$




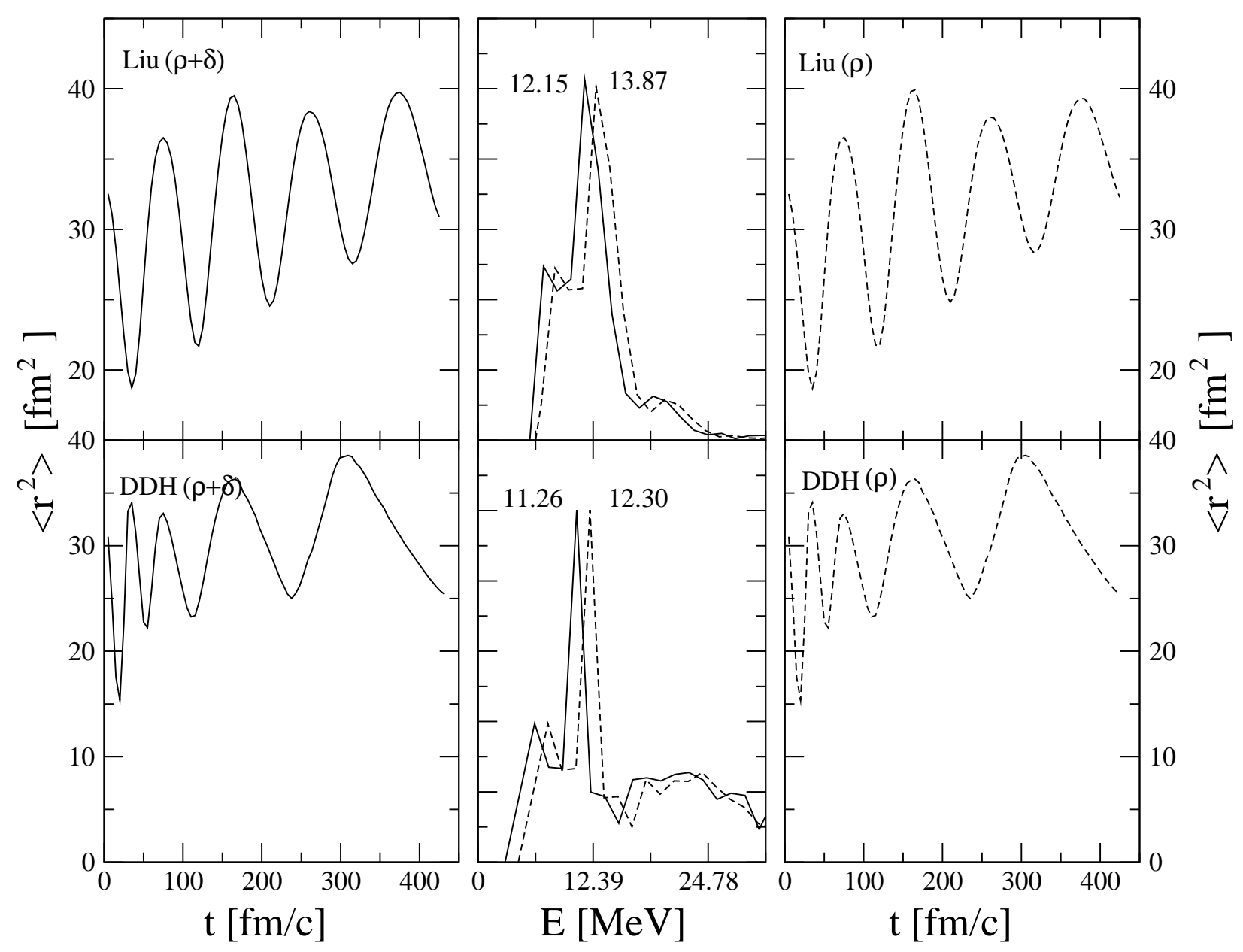

FIG. 2: (Left and right pannels) Time-dependent isoscalar $\left\langle r^{2}\right\rangle$ monopole moment and (middle pannels) the corresponding Fourier power spectra for ${ }^{208} \mathrm{~Pb}$. Transport calculations with the parameter sets are shown: (panels on the top) Liu - RMF with $(\rho)$ dashed line (right) and with $(\rho+\delta)$ solid line (left). (panels on bottom) Density Dependent model $(D D H)$ (lower panel) with $(\rho)$ dashed line (right) and with $(\rho+\delta)$ solid line (left). The excitation energy of $I S G M R$ for each parameter set is explicitly indicated in the Fourier power spectra and for comparison the results with $(\rho+\delta)$ and $(\rho)$ for both parameter set are shown together.

with $I \equiv\left(\rho_{n}-\rho_{p}\right) / \rho$, asymmetry parameter. We note the interplay between slope, $L \equiv$ $3 \rho_{0} \epsilon_{\text {sym }}^{\prime}(\rho)$, and curvature, $K_{\text {sym }} \equiv 9 \rho_{0} \epsilon_{\text {sym }}^{\prime \prime}(\rho)$ of the symmetry energy at saturation. The asymmetric matter becomes softer since the shift is in general negative, due to the dominance of the slope term L 31], see 2] and the recent discussion in 32]. Thus, in ${ }^{208} \mathrm{~Pb}, \mathrm{n}$-rich system, $I=0.21$, asymmetry can affect the the isoscalar monopole oscillations, as also noted in refs. 15, 16, 17] within relativistic and non-relativistic frames. 
In our NL3 vs. NL2 - G comparison the difference on the $a_{4}$ values, see Table II automatically implies a difference on the slope parameter $L$, since in both models the potential symmetry energy is coming from the effective $\rho$-meson coupling which leads to a linear $\rho_{B^{-}}$ dependence. So the larger $a_{4}$ of $N L 3$ means a larger slope at $\rho_{0}$ and so a larger reduction of the compressibility in ${ }^{208} \mathrm{~Pb}$, see Eq.(24). Our conclusion is that in NL3 both effects, smaller effective mass and larger $a_{4}$, are almost compensating the much larger $K_{n m}$ value, finally leading to a monopole frequency in ${ }^{208} \mathrm{~Pb}$ not much different from the $N L 2-G$ one.

We have continued the analysis of the symmetry contribution studying the effect of a scalar isovector channel with the inclusion of $\delta$ meson both in nonlinear and density dependent models. The results are shown in Fig 2. The time history of isoscalar monopole moment and its Fourier spectrum are shown for the Liu sets in the upper panel with isovector $\rho+\delta$ interaction $(N L \rho \delta$, solid line) and with $\rho$ interaction $(N L \rho$, dashed line) and similarly with density dependent models (lower panel). For both interactions although the time evolution shows a very similar behaviour the power spectrum presents a net reduction in the energy centroid when one includes the $\delta$ meson. This is a very nice indication of the dominance of the "Slope" of the symmetry energy on the compressibility shift. In fact when the $\delta$-channel is included we have a clear increase of the symmetry energy slope $L$ around saturation, see the discussion after Eq.(6-19) of ref. [2]; e.g. it results about $20 \%$ larger in the Liu parametrizations.

Our discussion suggests that although the inclusion of $\delta$ meson does not produce important effects on the ground state of finite nuclei [22], it has an interesting influence on the collective excitations of charge asymmetric finite nuclei, in particular for ${ }^{208} \mathrm{~Pb}$. This appears a good suggestion for new experiments aiming to a better determination of the poorly known slope (and curvature) of the symmetry term around saturation.

In general the $D D H$ monopole frequencies are systematically below the corresponding Liu ones. However this appears mostly a joint effect of different effective masses and symmetry energies, as already discussed for the NL3 vs. NL2-G comparison. Therefore the density dependence of the coupling constants seems not largely affecting the monopole response. In fact this can be expected from the rather smooth behaviour around $\rho_{0}$, see Fig.1 of ref. [22]. A more detailed study is presented in the Section V.

Finally we like to note again that, although the Liu's $N L \rho$ parametrization has a smaller $K_{n m}$ compared to $N L 3$, it produces roughly the same monopole main frequency due to a 
larger effective mass joint to a smaller $a_{4}$. Of course we cannot compare the $N L \rho \delta$ results since the $\delta$ channel is absent in $N L 3$.

\section{ISOVECTOR DIPOLE OSCILLATIONS}

In the literature only Relativistic $R P A$ calculations have been performed so far to study the well known isovector giant dipole resonance $(I V G D R)$ 14, 28]. It has been reported that for calculations of higher multipole modes, other than monopole, the response of a nucleus is difficult to evaluate in the time-dependent $R M F$ [14]. The reason for this is that, since rotational invariance is broken and the differential equations have to be explicitly solved at each time-step on a two dimensional mesh in coordinate space, it becomes difficult to keep the solutions stable for the very long times that are necessary for a good accuracy. The problem is overcome in the present $R B U U$ simulation, since it is possible to study the time history of $I V G D R$ in a time dependent frame as already done in the $I S G M R$ case.

The experimental IVGDR energy in ${ }^{208} \mathrm{~Pb}$ is well known as $13.5 \pm 0.2 \mathrm{MeV}[33]$. Since in the isovector channel we mainly want to study the effect of the inclusion of isovector-scalar couplings, we only present results of the models that are parametrized with and without $\delta$ meson. In Fig. 3 we present the ${ }^{208} \mathrm{~Pb} I V G D R$ oscillations and the corresponding Fourier transforms within models that include isovector-scalar channel namely Liu (upper panel) with $\rho+\delta$, solid line(left) and $\rho$, dashed line (right) and similarly for Density Dependent parameter sets (lower panel). The Fourier transforms show a good single frequency dominance of the isovector mode. We observe that the $D D H$ models, with $a_{4}=33.4 \mathrm{MeV}$, systematically give a larger resonance energy compare to Liu $-R M F$ sets, with $a_{4}=30.5$ $\mathrm{MeV}$. Moreover in both models a clear reduction of centroid energy is observed when the $\delta$-meson is included.

It is a well known fact that the dynamics of $I V G D R$ is rather sensitive to the symmetry energy of the corresponding model which is acting as a kind of restoring force parameter. The drawback in previous relativistic models is the one-to-one correspondence between $a_{4}$ and $I V G D R$ energy [14], and so it is difficult to discriminate among the different interactions. This is not the case in our analysis. The new important conclusion that can be drawn from our results is that the IVGMR dynamics is also sensitive to the more microscopic covariant structure of the symmetry term, i.e. to the interplay of various isovector channels. The 

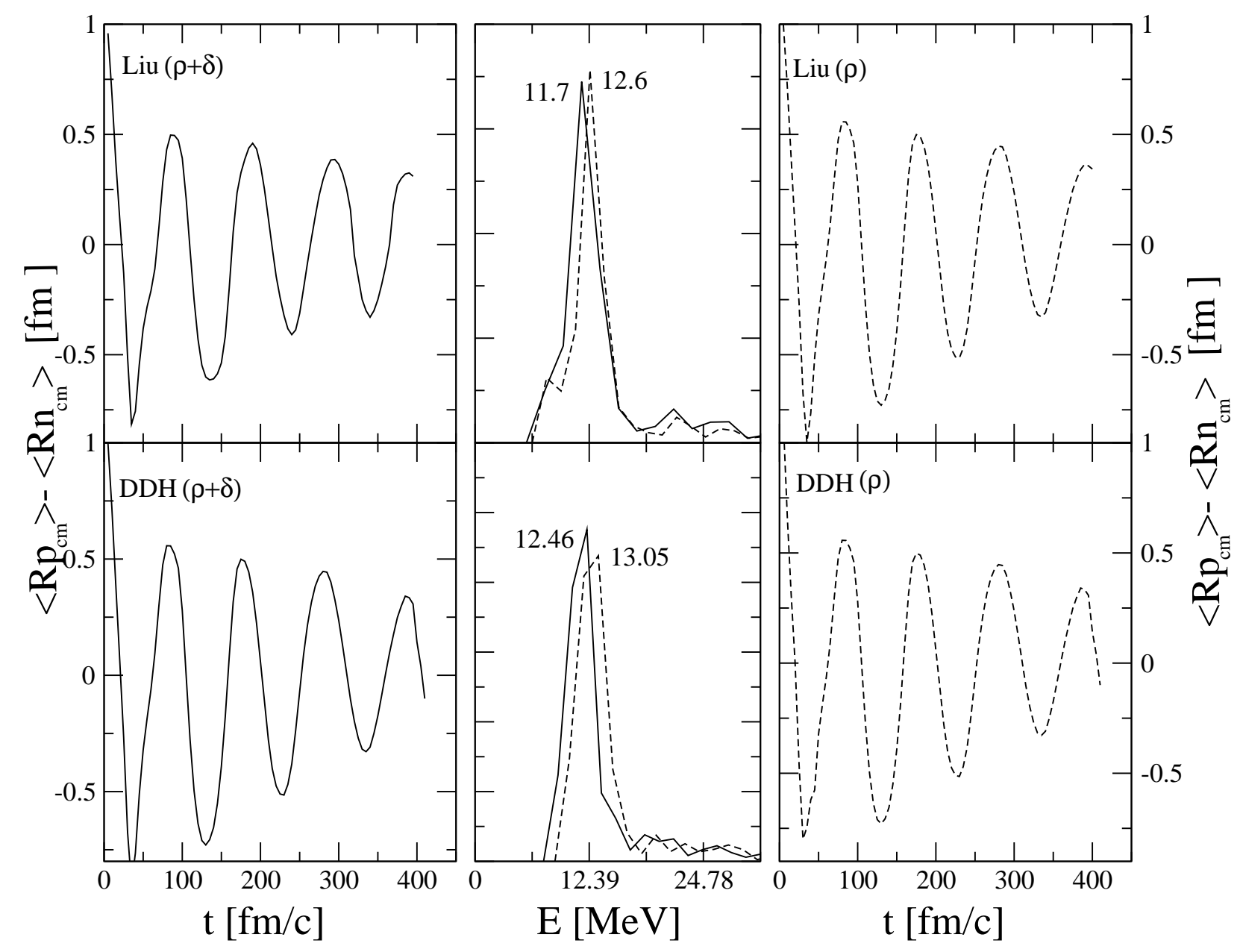

FIG. 3: Time-dependent isovector dipole moment (center of mass displacement of protons and neutrons $\left.\left\langle R p_{c m}\right\rangle-\left\langle R n_{c m}\right\rangle\right)$ and the corresponding Fourier power spectrum for ${ }^{208} \mathrm{~Pb}$. The parameter set of the effective Lagrangian is "Liu" (upper panel) with $(\rho)$ dashed line (right) and $(\rho+\delta)$ solid line (left), and Density Dependent model $(D D H)$ (lower panel) with $(\rho)$ dashed line (right) and $(\rho+\delta)$ solid line (left). The excitation energy of ISGMR for each parameter set is explicitly stated on the Fourier power spectrum and for comparison the results with $(\rho+\delta)$ and $(\rho)$ for both parameter sets are shown together.

physical interpretation of this result can be given in terms of the isovector response derived Ref.[3], where it is shown that the potential part of the symmetry energy explicitly appears in the dispersion relations with an correction term having a definite $f_{\rho}, f_{\delta}$ structure:

$$
1+\frac{6 E_{F}^{*}}{k_{F}^{2}}\left[E_{s y m}^{p o t}-\frac{f_{\rho}}{2} \frac{k_{F}^{2}}{E_{F}^{* 2}}\left(1-f_{\delta} \frac{m^{*}}{E_{F}^{* 2}} \rho_{S}\right) \rho_{B}\right] \varphi(s)=0
$$

Note the similarity with the corresponding dispersion relation for isoscalar modes Eq.(23), 
in particular the parallel role played by comressibility and symmetry energy in the two collective degrees of freedom.

Now we can easily have interactions with the same $a_{4}$ value at normal density but with rather different isovector dipole response. In fact when including the $\delta$ channel we have to increase the $f_{\rho}$ coupling in order to have the same symmetry energy at saturation ( $\delta$, scalar field, is attractive in the isospin degree of freedom, see the discussion in Sect.6 of ref.[2]). Then the isovector dipole "effective restoring force" (coefficient of the Lindhard function in the Eq.(25) will be reduced.

A similar effect has been pointed out in a detailed non-relativistic Skyrme-RPA study of the Giant Dipole Resonance in heavy nuclei $\left({ }^{208} \mathrm{~Pb}\right)$ using effective interactions with various isovector terms [34]. A separate sensitivity of the average resonance frequencies on the symmetry energy $a_{4}$ and on its slope has been found. In a covariant scheme we can see from Eq.(25) that such behaviour can be achieved only by using two isovector fields, at the lowest order. This result shows more generally that a dynamical observable can be more sensitive to the microscopic structure of the isovector interaction than static properties. For instance in a careful study of the neutron distributions, [35], it is clearly shown that these "equilibrium" observables are almost equally correlated to value, slope and curvature of the symmetry term.

Finally we see that even for the isovector dipole we cannot reveal specific contributions related to a density dependence of the coupling constants, in the sense that all the observed differences can be accounted for just in terms of symmetry energies and of isovector channels. We must say that we have not really performed an accurate analysis of this point, e.g. comparing with $R M F$ models with exactly the same saturation properties. Enlightening analitical results can be derived within a nuclear matter linear response theory, as shown in the following Section.

\section{NUCLEAR MATTER RESPONSE WITH DENSITY DEPENDENT VER- TICES}

In previous Sections in order to explore the relevant physical quantities affecting the energy centroid of the collective modes, we have compared results from various $Q H D$ models and parametrizations. Furthermore to have a guidance on the observed effects in finite nuclei, 
we took advantage of the plain dispersion relations for collective modes in nuclear matter studied in Ref. 3] in the contest of $Q H D-N L$ models. However in the $D D H$ approach, where meson-nucleon couplings depend on the vector (baryon) density, the relation between the coupling functions and the compressibility can be expected to be modified together with the dispersion relations for isoscalar and isovector modes.

By a comparison of results with the different parametrizations, we have argued that the density dependence of meson-nucleon couplings do not carry specific contributions at least to a large extent. In order to corroborate such a statement we briefly discuss the pertinent modifications of the thermodynamic quantities together with the linear response theory in nuclear matter, showing that in $D D H$ models most of the effect is reabsorbed by the compressibility and large corrections may be expected only for very strong density dependence of the couplings.

We remind that the $D D H$ model has all the meson-nucleon couplings dependent on the isoscalar vector (baryon) density, defined as $\varrho=\sqrt{j_{\mu} j^{\mu}}$, with $j_{\mu}$ defined as in Section II. Such a density dependence leads to rearrangement contributions that affect the vector self-energy adding a term that at mean field level reads as:

$$
\Sigma_{\mu}^{R}=\left(\frac{\partial f_{\omega}}{\partial \varrho} \varrho^{2}-\frac{\partial f_{\sigma}}{\partial \varrho} \rho_{s}^{2} \pm \frac{\partial f_{\rho}}{\partial \varrho} j_{3 \mu} j_{3}^{\mu} \mp \frac{\partial f_{\delta}}{\partial \varrho} \rho_{s 3}^{2}\right) \frac{j_{\mu}}{2 \varrho} .
$$

The effect of rearrangement terms on the pressure can be find in refs. [6, 7]. More relevant for our discussion is how they enter the compressibility:

$$
K=3 \frac{k_{F}^{2}}{E_{F}^{*}}+9\left(f_{\omega} \rho_{B}-f_{\sigma} \frac{m^{*}}{E^{*}} \frac{d \rho_{s}}{d \rho_{B}} \rho_{B}\right)+9\left(\frac{d f_{\omega}}{d \rho_{B}} \rho_{B}^{2}-\frac{d f_{\sigma}}{d \rho_{B}} \frac{m^{*}}{E^{*}} \rho_{s} \rho_{B}\right)+9 \frac{d \Sigma_{0}^{R}}{d \rho_{B}} \rho_{B}
$$

with

$$
\frac{d \Sigma_{0}^{R}}{d \rho_{B}}=\left(\frac{d f_{\omega}}{d \rho_{B}} \rho_{B}-\frac{d f_{\sigma}}{d \rho_{B}} \frac{d \rho_{s}}{d \rho_{B}} \rho_{s}\right)+\frac{1}{2}\left(\frac{d^{2} f_{\omega}}{d \rho_{B}^{2}} \rho_{B}^{2}-\frac{d^{2} f_{\sigma}}{d \rho_{B}^{2}} \rho_{s}^{2}\right)
$$

we can see that Eq.(27) reduces to the standard formula of $Q H D$ with costant couplings when the coupling are density indipendent, see Ref. [2].

The linear response in nuclear matter can be derived along the same lines of Ref.[3], but one as to redefine the vector self-energy $\Sigma_{\mu}$, defined in Eq.(8), adding the rearrangement term $\Sigma_{\mu}^{R}$ and consequently the effective kinetic momenta, defined in Eq.(10). Once also the equilibrium Wigner matrix is consistently redefined, on the same fashion of Ref. [3] a 
dispersion relation for isoscalar modes in symmetric nuclear matter can be written as:

$$
1+\frac{E_{F}^{*}}{3 k_{F}^{2}}\left[K_{n m}^{p o t}-9 f_{\omega} \frac{k_{F}^{2}}{E_{F}^{* 2}}\left(1-f_{\sigma} \frac{m^{*}}{E_{F}^{* 2}} \rho_{s}+\frac{\rho_{s}^{2}}{E_{F}^{*}} \frac{d f_{\sigma}}{d \rho_{B}}\right) \rho_{B}\right] \varphi(s)=0 .
$$

if terms associated with $\frac{\partial \rho_{s}}{\partial m^{*}}$ are discarded, or in other words, if the safe approximation $\frac{d \rho_{s}}{d \rho_{B}}=\frac{m^{*}}{E^{*}}$ is taken. We can see that of the derivative of the coupling function are reabsorbed in the $K_{n m}^{p o t}$. The difference between Eq.(23) and Eq.(29) is the last term. With some algebra, within the same approximation scheme, the contribution of density dependent terms can be reduced to a variation of the $\sigma$-coupling, from $f_{\sigma}$ to $f_{\sigma}-\frac{d f_{\sigma}}{d \rho_{B}} \rho_{B}$, in the correction term of Eq.(23). Due to the other quenching factors and to the smoothness of the $f_{\sigma}\left(\rho_{B}\right)$ function around $\rho_{0}$, we can expect an overall variation of a few percent in the dispersion relation.

We note however that now the $f_{\omega}\left(\rho_{0}\right)$ is not exactly the same of $Q H D-N L$ (with scalar non-linear terms), because the rearrangement terms affect also the relation for the binding energy, that in $D D H$ models is given by:

$$
E / A\left(\rho_{0}\right)=E_{F}^{*}+f_{\omega} \rho_{0}+\Sigma_{0}^{R}-m_{N}
$$

From Eq.(26) we see that $\Sigma_{0}^{R}$ is given by the difference in the density slope between the scalar and vector field. Therefore it may not be discarded in general, but is usually subdominant due to the similar density dependence of the scalar and vector coupling functions around saturation density, see Fig.1 of ref.[22].

The expression for the symmetry energy is not modified in $D D H$ models respect to $Q H D-N L[1]$, because the couplings depend only on the isoscalar vector density. Also the dispersion relation for the isovector mode in symmetric nuclear matter is not affected by the density dependence of the couplings and the relation written in Eq.(25) is still valid. However in asymmetric nuclear matter new terms appear, that are proportional to the asymmetry $I$ and to the derivative of the $\rho$-like and $\delta$-like couplings. Therefore in $D D H$ models for exotic nuclei there is the possibility to have a modified relation between symmetry energy and dipole excitation, however an exhaustive study of such effects goes beyond the scope of this paper. 


\section{CONCLUSIONS}

Aim of this work has been to study effects on collective excitation properties of asymmetric spherical nuclei of the detailed structure of the nuclear $E o S$ in the isoscalar and isovector sector. Particular attention has been put on the dynamical implications of the inclusion of a scalar isovector contribution due to an effective $\delta$ meson coupling.

We have described the dynamics of the isoscalar monopole and isovector dipole oscillations in a relativistic transport approach based on a nucleon-meson effective field interaction. We have evaluated the time-evolution of the oscillations obtaining then the corresponding excitation energies from the power spectra of the modes. The applications are to the n-rich nucleus ${ }^{208} \mathrm{~Pb}$, using a suitable choice of different effective Lagrangians.

For the monopole mode we show an interesting $m^{*}$, effective mass, dependence of the centroid energy. This effect, joint to a symmetry energy contribution, can account for the claimed ambiguity on the difference in compressibility moduli, that produce the experimental energy of $I S G M R$ in ${ }^{208} \mathrm{~Pb}$, between relativistic and nonrelativistic microscopic models. From the influence of the $\delta$ meson, just a systematic reduction of the peak energy, we have inferred that the symmetry energy effect on the isoscalar monopole is mainly due to variations of the "Slope" $-L$ around saturation. This is an important result, since it could open the possibility of a direct access to this poorly known parameter from monopole data changing the neutron number in a fixed isotope. Moreover we will be able to trace back the covariant structure of the effective interaction in the isovector channel, of relevance also for relativistic heavy ion collisions [22].

The isovector dipole response is directly linked to the isospin dependent part of the nuclear $E_{o S}$. The new result shown here is that the IVGDR energy is decreasing when the $\delta$ coupling is included, keeping fixed the symmetry energy at saturation. The effect can be easily understood from the form of isovector dispersion relations in nuclear matter in a relativistic linear response approach. We can then have different $I V G D R$ energies for effective interactions that show the same $a_{4}$ value, but with a different covariant field structure in the isovector channel. We note the similarity to the isoscalar case, just exchanging the roles of compressibility and isoscalar couplings with symmetry energy and isovector couplings.

Finally we do not see large effects from the Density Dependence of the effective meson couplings. This is mainly due to the smooth behavior around $\rho_{0}$. Moreover we have shown 
that some compensation is also coming from dynamical contributions of the rearrangement terms.

\section{Acknowledgments}

S.Y would like to thank the staff of Laboratori Nazionali del Sud (LNS), INFN, in Catania

for the hospitality during his visit to LNS. This work has been supported in part by Mersin University under the contact No. BAB FEF FB (SY) 2004-2. 
[1] B.Liu, V.Greco, V.Baran, M.Colonna, M.Di Toro, Phys.Rev. C65, 045201 (2002).

[2] V.Baran, M.Colonna, V.Greco, M.Di Toro, Phys.Rep. 410, 335-466 (2005).

[3] V.Greco, M.Colonna, M.Di Toro, F.Matera, Phys.Rev. C67, 045201 (2003).

[4] P.Ring, D.Vretenar and B.Podobnik, Nucl.Phys. A598, 107 (1996).

[5] G.A.Lalazissis, J.Konig and P.Ring, Phys.Rev. C55, 540 (1997).

[6] C.Fuchs, H.Lenske, H.H.Wolter, Phys.Rev. C52, 3043 (1995).

[7] S.Typel, H.H.Wolter, Nucl.Phys. A656, 331 (1999).

[8] M.V.Stoitsov, P.Ring, and M.M.Sharma, Phys.Rev. C50, 1445 (1994).

[9] D.Vretenar, N.Paar, P.Ring, and T.Niksic, Phys.Rev. C65, 021301 (2002).

[10] D.Vretenar T.Niksic, and P.Ring, Phys.Rev. C68, 024310 (2003).

[11] J.P.Blaizot, Phys.Rep. 64, 171 (1980).

[12] J.P.Blaizot, J.F.Berger, J.Decharge and M.Girod, Nucl.Phys. A591, 435 (1995).

[13] D.Vretenar, G.A.Lalazissis, R.Behnsch, W.Poschl and P.Ring, Nucl.Phys. A621, 853 (1997).

[14] Z.Y.Ma, N.Van Giai, A.Wandelt, D.Vretenar and P.Ring, Nucl.Phys. A686, 173 (2001).

[15] J.Piekarewicz, Phys.Rev. C66, 034305 (2002).

[16] B.K.Agrawal, S.Shlomo, V.Kim Au, Phys.Rev. C68, 031304(R) (2003).

[17] G.Coló, N.Van Giai, J.Meyer, K.Bennaceur and P.Bonche Phys.Rev. C70, 024307 (2004).

[18] B.D.Serot,J.D.Walecka, Adv.Nucl.Phys. 16, 1 (1986).

[19] W.Botermans and R.Malfliet, Phys.Rep. 198, 115 (1990).

[20] B.Blaettel, V.Koch, U.Mosel, Rep.Prog.Phys. 56, 1 (1993).

[21] P.Danielewicz, Ann.Phys. 152, 239 (1984).

[22] T.Gaitanos, M.Di Toro, S.Typel, V.Baran, C.Fuchs, V.Greco, H.H.Wolter, Nucl.Phys. A732, $24(2004)$.

[23] T.Maruyama, W.Cassing, U.Mosel, S.Teis, K.Weber, Nucl.Phys. A573, 653 (1994).

[24] C.Fuchs, H.H.Wolter, Nucl.Phys. A589, 732 (1995).

[25] H.Stöcker, W.Greiner, Phys.Rep. 8, 137-277 (1986).

[26] S.Shlomo and D.H.Youngblood, Phys.Rev. C47, 52 (1993).

[27] D.Vretenar, N.Paar, P.Ring, G.A.Lalazissis, Nucl.Phys. A692, 496 (2001).

[28] T.Niksic, D.Vretenar, P.Ring, Phys Rev. C66, 064302 (2002). 
[29] B.Podobnik, D.Vretenar and P.Ring, Z.Phys. A354, 375 (1996).

[30] A.Smerzi, A.Bonasera, M.Di Toro, Phys.Rev. C44, 1713 (1991).

[31] In fact the symmetry energy curvature is also negative for most effective interactions (we have also a negative contribution from the kinetic part). It can be slightly positive only for a highly increase, of parabolic type, of the potential contribution around the saturation point, ref. [2].

[32] L.-W.Chen, C.M.Ko, B.-A.Li, Phys.Rev.Lett. 94, 032701 (2005).

[33] B.L.Berman and S.C.Fultz, Rev.Mod.Phys. 47, 713 (1975).

[34] P.G.Reinhard, Nucl.Phys. A649, 305c (1999).

[35] R.J.Furnstahl, Nucl.Phys. A702, 85 (2002). 